\title{
In Vivo Potential of Manganese Chelated Porphy- somes as MRI Contrast Agents
}

\author{
AUTHORS: Chris J. Zhang ${ }^{1}$, Michael S. Valic ${ }^{2}$, Juan Chen ${ }^{3}$, Gang Zheng ${ }^{1,2,3}$ \\ SCHOOL/INSTITUTIONAL AFFILIATION: \\ 1- Department of Medical Biophysics, University of Toronto \\ 2- Institute of Biomaterials and Biomedical Engineering, University of Toronto \\ 3- Techna Institute for the Advancement of Technology for Health, University Health Network
}

10.17975/sfj-2017-007

\section{Corresponding author: Gang Zheng (gang.zheng@uhnresearch.utoronto.ca)}

\section{Abstract}

Porphysome nanoparticles are composed of porphyrin-conjugated lipids. The attachment of the porphyrin moiety to each phospholipid confers novel properties to the liposome-like nanoparticle, allowing it to perform a variety of diagnostic and therapeutic applications. The metal chelating properties of porphyrin can be used to bind manganese $(\mathrm{Mn})$, transforming the porphysome into a contrast agent for magnetic resonance imaging (MRI). Previous work has extensively characterized the properties of the Mn-porphysome. Herein, we build upon that work by demonstrating the bio-interactions of Mn-porphysomes in vitro to validate their study in vivo. Particle stability in serum was inferred from fluorescence quenching efficiency, and tolerability to cells was measured using an MTT assay. Mn-porphysomes remained $>80 \%$ quenched after $14 \mathrm{H}$ and showed no toxicity to cells at concentrations below $125 \mathrm{mM}$. These preliminary results suggest that the porphysome may be used to enhance MRI contrast in vivo.

\section{Key Words}

Porphysome, MRI contrast agent, Manganese, Nanoparticles, Porphyrin

\section{Introduction}

The porphysome is a liposome-like nanoparticle made from porphyrin-lipid monomers with diverse potential for cancer nanomedicine. The unique conjugation of results in a combination of properties of liposomes (phospholipids) and porphyrins, as well as some emerging properties resulting from synergistic interactions. The porphysome has many applications for cancer nanomedicine including the ability to act as a fluorophore for tumour delineation [1], activatable photosensitizer [2], photothermal agent [3], and radiotracer [4].

The porphysome also has the potential to act as a contrast agent for magnetic resonance imaging (MRI), but this remains to be explored. MRI is a non-invasive, non-ionizing, high-resolution imaging modality that can be enhanced through the use of contrast agents, which function by locally affecting the magnetic moments of hydrogen nuclei. Manganese $(\mathrm{Mn})$ is a paramagnetic metal, and its permanent net dipole allows it to exert a $T_{1}$ and $T_{2}$ shortening effect. Free ionic manganese cannot be used due to issues with neurodegenerative toxicity and poor bioavailability [5]. These barriers can be overcome by the stable chelation of the metal to the porphyrin moiety of porphyrin-lipid to create Mn-porphyrin-lipid. The nanoparticles formed from Mn-porphyrin-lipid monomers have different properties from free-base (non-chelated) porphysomes. In addition to paramagnetic effects conferred by Mn, the Mn-porphysome is non-photobleachable, displays quenching of fluorescence even in its monomeric form, and exhibits only non-radiative relaxation following excitation [6].

Previous work has extensively characterized the MR properties of Mn-porphysomes in vitro and reported $T_{1}$ and $T_{2}$ relaxivity values $\left(r_{1}\right.$ and $\left.r_{2}\right)$ of $1.2 \mathrm{mM}^{-1} \mathrm{~s}^{-1}$ and $7.0 \mathrm{mM}^{-1} \mathrm{~s}^{-1}$ respectively [7]. This study builds on previous work by focusing on the bio-interactions of these particles. These preliminary results enable the study of the Mn-porphysome as an MRI contrast agent for enhanced tumour detection in vivo, which contributes to the inherent multimodality of the porphysome. Nano-sized MRI contrast agents may help increase the limit of detection, which can aid tumour screening and lead to early intervention.

\section{Materials and Methods}

\section{Manganese chelation}

A solution of 1 molequivalentporphyrin-lipid (pyropheophorbide- $\alpha$ conjugated to 1-palmitoyl-2-hydroxy-sn-glycero-3-phosphocholine), $5 \mathrm{~mol}$ equivalent $\mathrm{MnCl}_{2}, 20 \mathrm{~mol}$ equivalent $\mathrm{NaOAc}$ in glacial acetic acid at a concentration of $4 \mathrm{mM}$ porphyrin-lipid was heated under reflux for 2 hours at $60^{\circ} \mathrm{C}$. The solvent was dried using a centrifugal evaporator and dissolved in chloroform. A liquid-liquid 
extraction was performed using 1:9 $\mathrm{MeOH}: \mathrm{H} 2 \mathrm{O}$ as the aqueous phase. Mn-porphyrin-lipid was retained in the organic phase.

\section{Particle formulation}

Mn-porphysome nanoparticles were composed of $40 \mathrm{~mol} \%$ Mn-porphyrin-lipid, $15 \mathrm{~mol} \%$ free-base porphyrin-lipid, $40 \mathrm{~mol} \%$ cholesterol (Spectrum) and $5 \mathrm{~mol} \%$ 1,2-distearoyl-sn-glycero3-phosphoethanolamine-N-[methoxy(polyethylene glycol)-2000] (CordenPharma). Components were prepared in chloroform and dried to a lipid film under a stream of nitrogen gas. Lipid films were hydrated using deoxygenated HEPES buffer ( $\mathrm{pH}$ 7.4). Solution was sonicated for 30 minutes at $60^{\circ} \mathrm{C}$ in a $40 \mathrm{kHz}$ bath sonicator (Branson 2510).

\section{Particle characterization}

Chelation of manganese by porphyrin-lipid was monitored using reverse-phase analytical UPLC-MS performed on a ACQUITY UPLC CSHTM Phenyl-Hexyl 1.7uM column, using a Waters uPLC system with a photodiode array detector, and a Waters TQ mass detector using electrospray ionization. The conditions were as follows: solvent A) $0.1 \%$ formic acid in water, and solvent $B$ ) $0.1 \%$ formic acid in acetonitrile; gradient, from $60 \% A+40 \% B$ to $100 \% B$ in the first 3 min, keeping $100 \%$ B for $1 \mathrm{~min}$, then to $60 \% A+40 \%$ B in 0.1 min and keeping for $2 \mathrm{~min}$; flow rate $0.6 \mathrm{~mL} / \mathrm{min}$. Particle size was characterized by dynamic light scattering using a Zetasizer ZS90 (Malvern).

\section{Serum stability}

$0.013 \mathrm{mM}$ free-base porphyrin-lipid prepared in a $60 \mathrm{v} / \mathrm{v} \%$ solution of fetal bovine serum. Fluorescence quenching efficiency of the particles was measured against identical samples disrupted with 0.5 v/v \% Triton X-100. Fluorescence measurements were performed using a CLARIOstar (Mandel) plate reader once every hour for 14 hours while incubated at $37^{\circ} \mathrm{C}$. Porphysomes were excited at 410 $\mathrm{nm}$ and the area under the curve (AUC) of the emission spectrum from $600-750 \mathrm{~nm}$ was calculated to compare the fluorescence intensity between Mn-porphysomes and the extent of fluorescence activation upon nanostructure dissociation.

\section{Particle tolerability to cells}

MAT-LyLu cells were chosen for their known biocompatibility with the Copenhagen rat strain, which will be used for in vivo studies. 2000 MAT-LyLu cells/well were seeded in a 96 well plate using RPMI 1640 medium and incubated at $37^{\circ} \mathrm{C}$. 24 hours after seeding, the cells were incubated with $200 \mu \mathrm{L}$ of either medium containing treatment or control untreated medium at $37^{\circ} \mathrm{C}$. After 24 hours, medium for different treatments was removed and replaced with medium containing $0.5 \mathrm{mg} / \mathrm{mL}$ MTT Reagent (3-(4,5-Dimethylthiazol-2-yl)-2,5-Diphenyltetrazolium Bromide, Invitrogen) and incubated for 2 hours at $37^{\circ} \mathrm{C}$. MTT reagent medium was removed and replaced with $100 \mu \mathrm{L}$ DMSO and incubated at room temperature for 2 hours. Absorbance was measured at $540 \mathrm{~nm}$ using a SpectraMlax M2 plate reader (Molecular Devices).

\section{Results}

We were able to synthesize and characterize manganese chelated, nano-sized porphysomes. The Mn-porphyrin-lipid monomers were identified by uPLC-MS. The expected $\mathrm{m} / \mathrm{z}$ for our monomer $\left(\mathrm{C}_{57} \mathrm{H}_{80} \mathrm{~N}_{5} \mathrm{O}_{9} \mathrm{PMn}\right)$ was 1065.2; we observed a molecular ion [M]+• of $1065.1 \mathrm{~m} / \mathrm{z}$, and a [M] ${ }^{2+\bullet}$ of $533.2 \mathrm{~m} / \mathrm{z}$ (Fig. 1A). Using dynamic light scattering to characterize the porphysome nanoparticles formed from these monomers, we observed a z-average size of $93.2 \pm 0.2$ $\mathrm{nm}$ and a polydispersity index of 0.130 (Fig. 1B). The Mn-porphysome's $T_{1}$ and $T_{2}$ relaxivity $\left(r_{1}\right.$ and $\left.r_{2}\right)$ have been previously reported to be $1.2 \pm 0.2 \mathrm{mM}^{-1} \mathrm{~s}^{-1}$ and $7.0 \pm 0.5 \mathrm{mM}^{-1} \mathrm{~s}^{-1}$ respectively [7].

Efficiency of porphysome fluorescence quenching is used to evaluate nanoparticle intactness because only intact porphysome nanostructures display fluorescence quenching. Mn-porphysomes show a decrease in quenching efficiency from 0.98 to 0.83 over a 14 hour period in $60 \mathrm{v} / \mathrm{v} \%$ fetal bovine serum at $37^{\circ} \mathrm{C}$ (Fig. $2 \mathrm{~A}$ ). The reduction of quenching efficiency is greater for Mn-porphysomes than free-base porphysomes which show a decrease in quenching efficiency from 0.98 to 0.93 over the same period.

Mn-porphysomes were tested for their tolerability to cells across a range of concentrations estimated to be physiologically relevant based on calculations of expected \% injected dose/gram accumulation at tumour. Mn-porphysomes display toxicity to MAT-LyLu cells at concentrations above $125 \mathrm{mM}$ as measured by an MTT assay (Fig. 2B). Both components of the Mn-porphysomes (free-base porphysomes and $\mathrm{MnCl}_{2}$ ) were found to be non-toxic across the same range. However, the observed difference in toxicity between Mn-porphysomes and controls above $125 \mathrm{mM}$ was not statistically significant $(p>0.05)$.

\section{Discussion}

Mn-porphysomes are manganese chelated nanoparticles, which makes them ideal candidates to act as MRI contrast agents. Their nano-size $(\leq 100 \mathrm{~nm})$ is suited for preferential tumour accumulation. 
A)

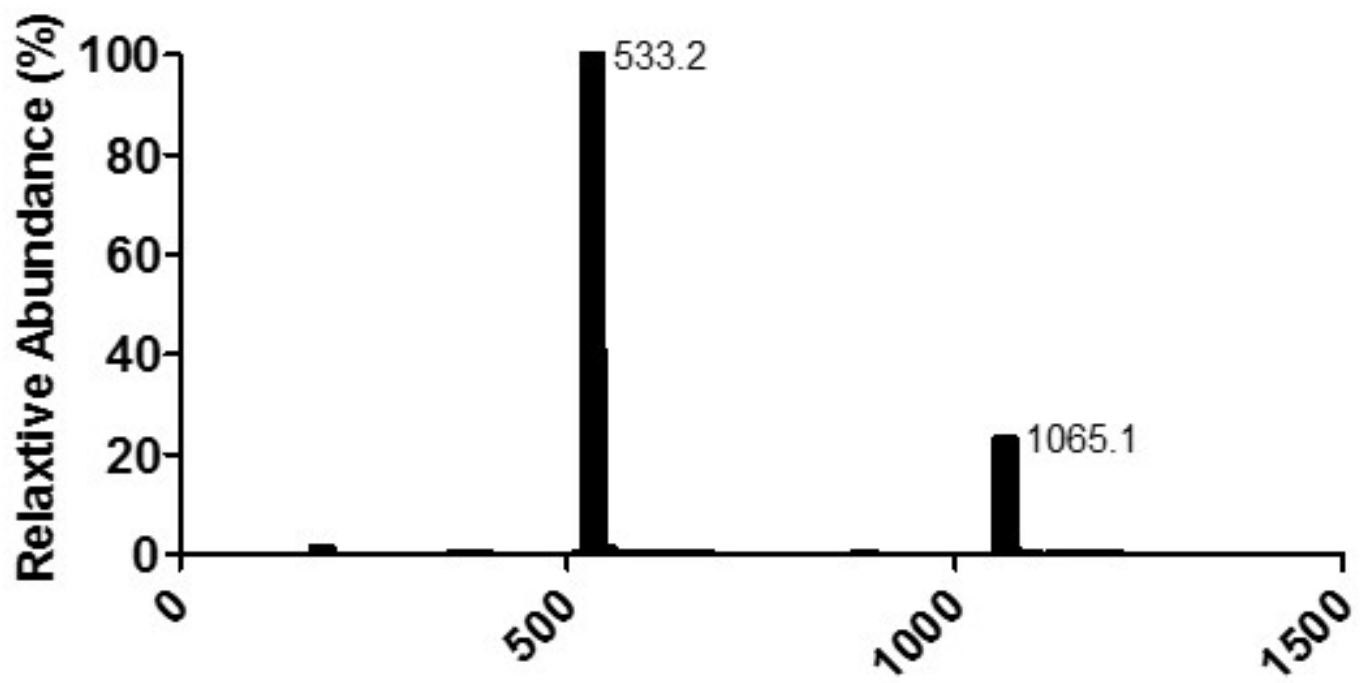

B)

$\mathrm{m} / \mathrm{z}$

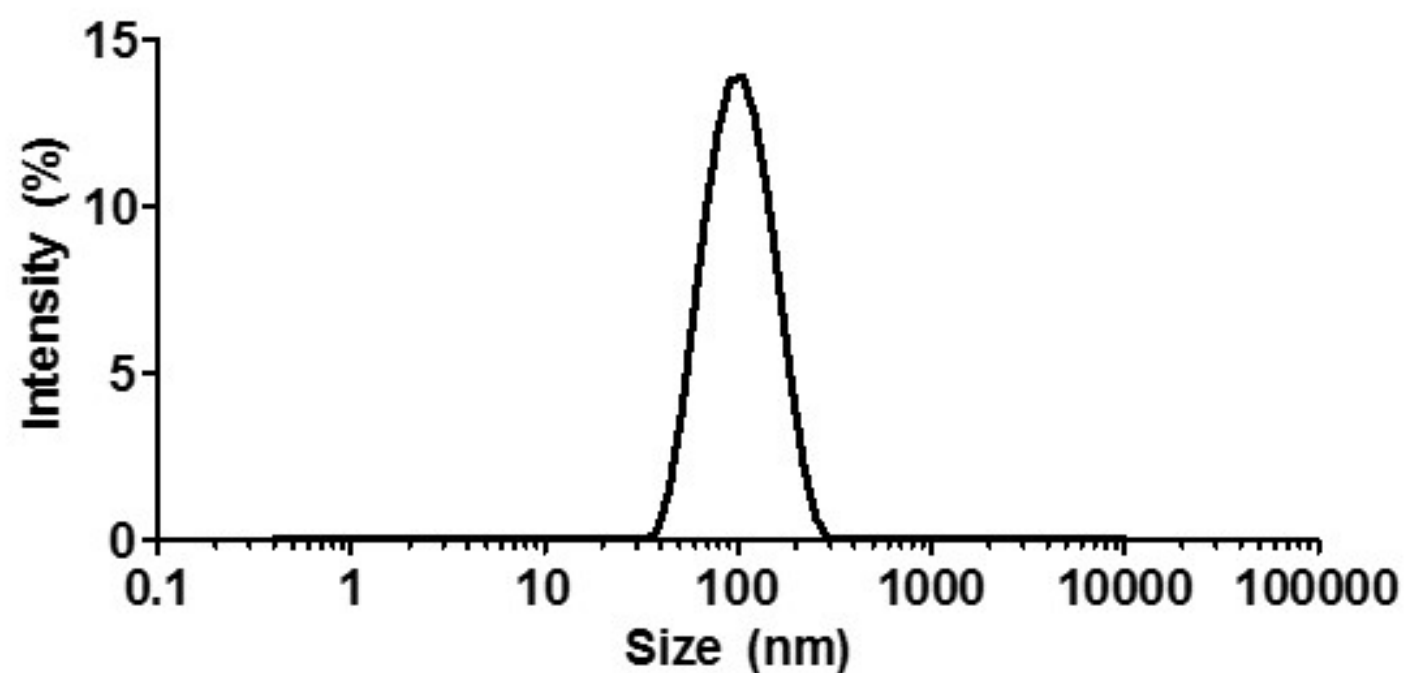

Figure 1: Characterization of Mn-porphysome nanoparticles.

A) Mass spectrum of Mn-porphyrin-lipid monomers. Molecular ion at $1065.1 \mathrm{~m} / \mathrm{z}$ closely matches theoretical sum of manganese and free-base porphyrin-lipid (1065.2 m/z). B) Representative particle size distribution by intensity determined using dynamic light scattering. Z-average size $=93.2 \pm 0.2 \mathrm{~nm}, \mathrm{PDI}=0.130$.

The Mn-porphysome is small enough to avoid significant detection by the mononuclear phagocytic system [8], but large enough to avoid renal filtration [9], thus allowing for prolonged circulation times and greater probability of extravasation to the tumour site. This is believed to occur by a mechanism called the enhanced permeability and retention (EPR) effect [10], though the ubiquity of this mechanism and the role of other factors has made it a controversial topic in recent years [11,12].

Stability in serum is important for nanoparticles to achieve long circulation times to facilitate tumour accumulation. Adsorption of, and interactions with serum proteins can destabilize the porphy- some, which will alter pharmacokinetics. We infer particle stability from quenching efficiency because the fluorescent porphyrin moieties demonstrate fluorescence quenching as a result of tight packing and interactions between molecules when assembled as a porphysome. Fluorescence is recovered upon dissociation into monomers. Particles that are not stable in serum display rapid decay of quenching efficiency [13].

We believe particle stability to be inversely related to Mn content. Chelation of Mn may limit the packing of monomers in the porphyrin bilayer, resulting in the observed decreased serum stability of Mn-porphysomes relative to free-base porphysomes. Particle 
A)

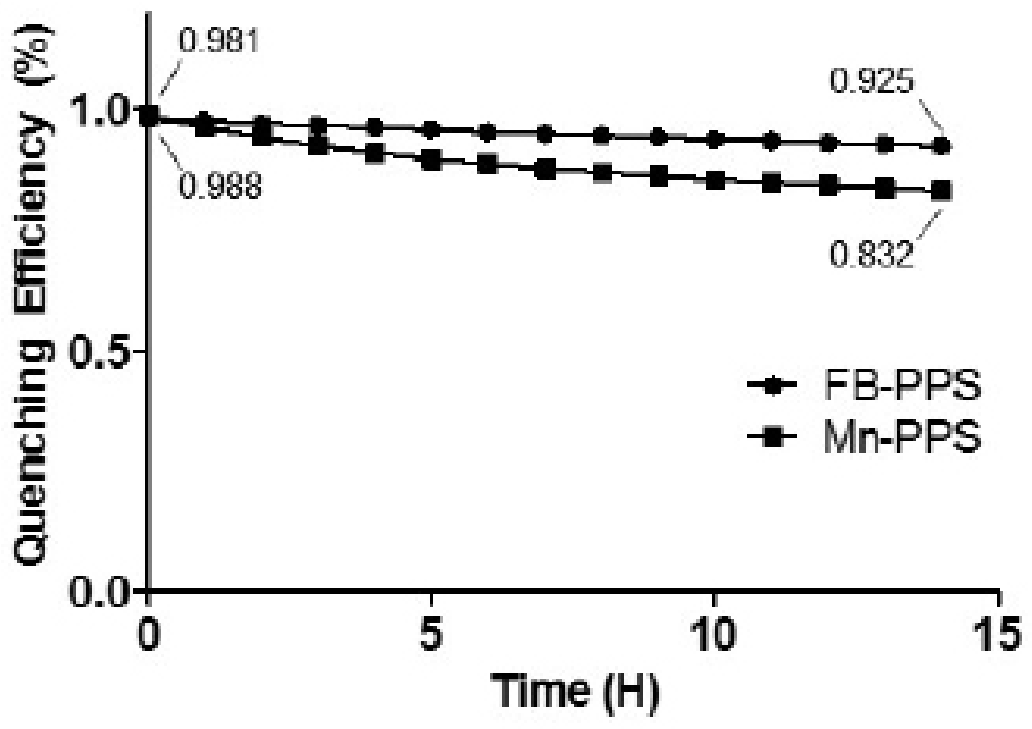

B)

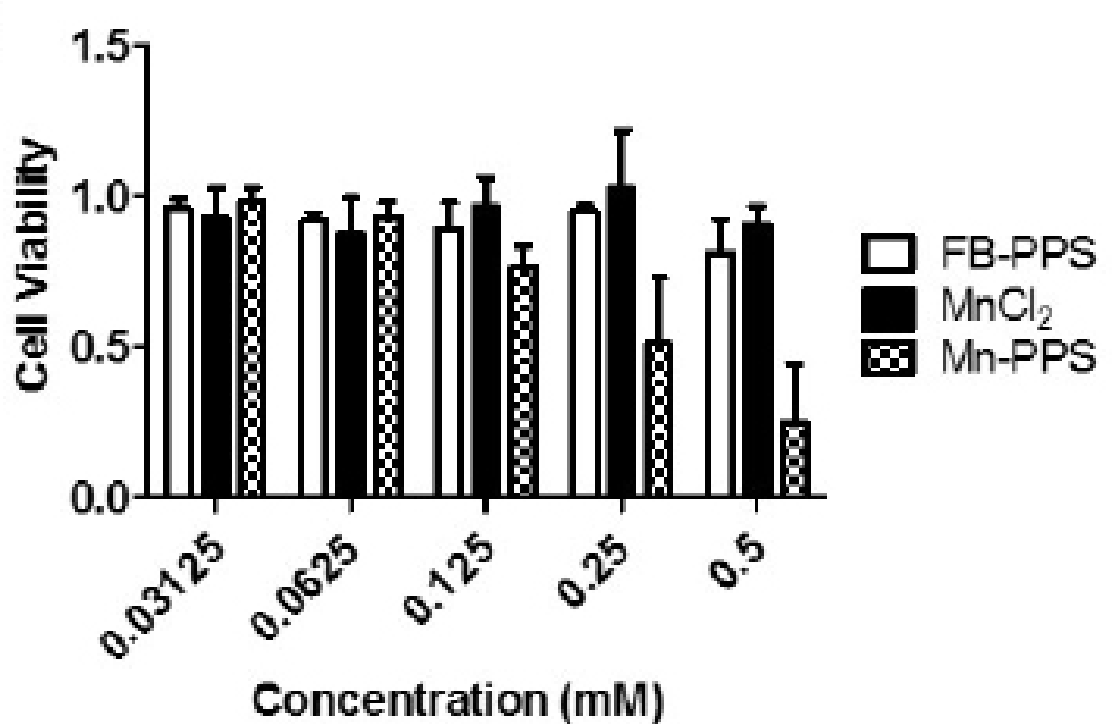

Figure 2: Bio-interactions of Mn-porphysome nanoparticles.

A) Stability of free-base porphysomes (FB-PPS) and Mn-porphysomes (Mn-PPS) in $60 \mathrm{v} / \mathrm{v} \%$ fetal bovine serum over a 14 hour period (mean of replicates, SD error bars, $\mathrm{n}=1$ ). Fluorescence quenching efficiency of porphysome nanoparticles used as a measure of particle structural stability. B) Viability of MAT-LyLu rat prostate tumour cells following treatment with various groups (mean, SEM error bars, $\mathrm{n}=3$ ). Viability determined using an MTT assay measuring absorbance at $540 \mathrm{~nm}$.

stability may be improved by increasing free-base porphyrin lipid, but high Mn content is desirable to provide greater MRI contrast. An optimal formulation must balance these two factors.

The chelation of $\mathrm{Mn}$ to the porphysome nanoparticle is able to intrinsically overcome the dopamine-dependent neurodegenerative effects $[14,15]$ since nanoparticles cannot readily cross the bloodbrain barrier [16]. Our study focused on a dopamine-free system to study particle tolerability to cells. Mn-porphysomes were an- ticipated to be completely tolerable, so the observed toxicity was surprising despite lack of statistical significance. Both free-base porphysomes and free $\mathrm{MnCl}_{2}$ did not show toxicity, which suggests the observed Mn-porphysome toxicity was likely a synthetic artefact due to excess reagents from chelation reaction. We hypothesize that this challenge can be overcome with additional purification.

Future work should be focused on investigating alternative methods to purify the Mn-porphyrin-lipid following chelation and con- 
firming the recovery of cell viability. Once this is established, the Mn-porphysome can proceed in vivo to validate its ability to act as an MRI contrast agent for tumour detection.

\section{Conclusions}

Appropriate bio-interactions are necessary for nanoparticles to function in vivo. The preliminary results of this study suggest that Mn-porphysome is stable in serum for $14 \mathrm{H}$ and is tolerable to cells up to $125 \mathrm{mM}$. These results complement the previous characterization of Mn-porphysomes and affirm the need for future in vivo studies. The limitation of particle purity may confound in vitro particle tolerability results and requires additional refinement to the synthesis and purification of the particle before use in vivo. This work helps to validate the use of the porphysome as a contrast agent

\section{References}

1. Liu TW, Macdonald TD, Jin CS, Gold JM, Bristow RG. Inherently Multimodal Nanoparticle-Driven Tracking and Real-Time Delineation of Orthotopic Prostate Tumors and Micrometastases. 2013;7(5):4221-32.

2. Jin CS, Cui L, Wang F, Chen J, Zheng G. Targeting-triggered porphysome nanostructure disruption for activatable photodynamic therapy. Adv Healthc Mater. 2014;3(8):1240-9.

3. Jin CS, Lovell JF, Chen J, Zheng G. Ablation of hypoxic tumors with dose-equivalent photothermal, but not photodynamic, therapy using a nanostructured porphyrin assembly. ACS Nano. 2013;7(3):2541-50.

4. Liu TW, MacDonald TD, Shi J, Wilson BC, Zheng G. Intrinsically copper-64-labeled organic nanoparticles as radiotracers. Angew Chemie - Int Ed. 2012;51(52):13128-31.

5. Pan D, Schmieder A, Wickline S, Lanza G. Manganese-based MRI contrast agents: past, present and future. Tetrahedron [Internet]. 2011;67(44):8431-44. Available from: http://www.sciencedirect.com/science/article/pii/S0040402011011409

6. Macdonald TD, Macdonald TD. Metalloporphysomes : Engineering New

Metalloporphyrin Nanoparticles by Metalloporphysomes. 2013;

7. Macdonald TD, Liu TW, Zheng G. An MRI-sensitive, non-photobleachable porphysome photothermal agent. Angew Chemie - Int Ed. 2014;53(27):6956-9.

8. Chen Z (Georgia). Small-molecule delivery by nanoparticles for anticancer therapy. 2010;16(12):594-602.

9. Choi HS, Liu W, Misra P, Tanaka E, Zimmer JP, Itty Ipe B, et for MRI, which will contribute to the inherent multimodality of this particle.

\section{Acknowledgements}

Thank you to Dr. Christina MacLaughlin for her discussion and guidance throughout this project. Also thanks to Dr. Kara Harmatys, Dr. Warren Foltz, Dr. Wenlei Jiang, and Danielle Charron for assistance with experiments. This work was funded by the Department of Medical Biophysics Summer Student Program (U of T). This work was presented as a talk at the Undergraduate Engineering Research Day 2016 (U of T), and as a poster at the Medical Biophysics Summer Poster Day 2016 (U of T).

al. Renal clearance of nanoparticles. Nat Biotechnol [Internet]. 2007;25(10):1165-70. Available from: http://dx.doi.org/10.1038/ nbt1340

10. Matsumura Y, Maeda H. A new concept for macromolecular therapeutics in cnacer chemotherapy: mechanism of tumoritropic accumulatio of proteins and the antitumor agents Smancs. Cancer Res. 1986;46(December):6387-92.

11. You Han Baea. Kinam Parkb. Targeted drug delivery to tumors: Myths, reality and possibility You. J Control Release. 2012;153(3):198-205.

12. Wilhelm S, Tavares AJ, Dai Q, Ohta S, Audet J, Dvorak HF, et al. Analysis of nanoparticle delivery to tumours. Nat Rev Mater [Internet]. 2016;1(5):16014. Available from: http://www.nature. com/articles/natrevmats201614

13. Ng KK, Takada M, Jin CCS, Zheng G. Self-sensing porphysomes for fluorescence-guided photothermal therapy. Bioconjug Chem. 2015;26(2):345-51.

14. Prabhakaran K, Ghosh D, Chapman GD, Gunasekar PG. Molecular mechanism of manganese exposure-induced dopaminergic toxicity. Brain Res Bull. 2008;76(4):361-7.

15. Crossgrove J, Zheng W. Manganese toxicity upon overexposure. NMR Biomed. 2004;17(8):544-53.

16. Shilo M, Sharon A, Baranes K, Motiei M, Lellouche J-PM, Popovtzer $R$. The effect of nanoparticle size on the probability to cross the blood-brain barrier: an in-vitro endothelial cell model. J Nanobiotechnology [Internet]. 2015;13(1):19. Available from: http://www.jnanobiotechnology.com/content/13/1/19 


\section{by Dr. Anne Johnson,}

\section{Chemistry Program Director, Department of Chemistry \& Biology, Ryerson University}

This is an interesting report on using porphysome nanoparticles as contrast agents to improve MRI scans. The authors mention that the Mn-porphysomes are slightly more toxic than controls above $125 \mathrm{mM}$. However, they do not provide us with the context we need to understand this. What are the controls, and how are they showing whether the results are valid? What concentration of porphysome is required to observe improvement in MRI contrast? Is $125 \mathrm{mM}$ above or below the concentration needed to observe differences in the MRI?

With regards to this paper (Zhang et al.), yes, I am recommending it for publication after the comments/suggestions are addressed.

\section{Supplementary Information}

\section{Materials and Methods}

\section{Animal models}

All animal work was performed in adherence to the Animal Use Protocol approved by University Health Network. 5 million GFP-LucMAT-LyLu cells in $200 \mu \mathrm{L}$ RPMI 1640 medium was subcutaneously injected in the right hind limb flank of an adult male Copenhagen rat and allowed to grow for about 2 weeks. A dose of Mn-porphyrin-lipid equivalent to $5.5 \mu \mathrm{mol} / \mathrm{kg} \mathrm{Mn}$ was injected intravenously in PBS buffer and $30 \%$ DMSO as an excipient.

\section{MR Imaging}

For in vivo rat studies, scanning used the B-GA12 gradient coil insert and $7.2 \mathrm{~cm}$ inner diameter RF volume coil. Rats were positioned in prone orientation on a slider bed with $1.8 \%$ anaesthesia delivered via nose cone. Respiration was monitored using the SA Instruments system (Stony Brook, NY). $T_{1}$ and $T_{2}$ maps were acquired as stacks of $2 \mathrm{D}$ axial slices with matching geometric features (60 x 48 mm field-of-view, $200 \times 160$ matrix, $300 \times 300$ micron in-plane resolution, $1.5 \mathrm{~mm}$ slice thickness). $\mathrm{T}_{2}$ mapping used a multi-spin-echo technique ( 36 echoes, 12 ms echo time, echo times ranging from 12 to $432 \mathrm{~ms} ; 6$ second repetition time, 12 min scan time). $T_{1}$ mapping used a variable repetition time technique (8 repetition times of $500,750,1000,1500,2000,2500,3000,5000 \mathrm{~ms}$; $16 \mathrm{~min} 15 \mathrm{sec}$ ). $T_{2}$ and $T_{1}$ maps were generated using in-line image sequence analysis. $T_{1}$ and $T_{2}$ values within tumor regions-of-interest were evaluated using MIPAV software (National Institutes of Health, Bethesda, MD).

\section{Results}

Mn-porphyrin-lipid enhances tumour contrast on $T_{1}$ relaxation maps of a subcutaneous tumour after 4 hours. The $T_{1}$ effect disappears 24 hours post injection and tumour contrast returns to base- line. Mn-porphyrin-lipid displays no noticeable $T_{2}$ tumour enhancing effect.

\section{Discussion}

These preliminary results are the first exploration of Mn-porphyrin-lipid in vivo and demonstrate a proof of principle of Mn-porphyrin-lipid based nanoparticles. Injection of Mn-porphyrin-lipid monomers serves as a control for behaviour of completely dissociated particles in vivo. We believe the mechanism for monomer tumour accumulation is through the enhance permeability and retention effect [1]. Tumour enhancement at earlier time points (4H) and loss of enhancement at later times $(24 \mathrm{H})$ can be explained with our understanding of pharmacokinetics of Mn-porphyrin-lipid sized monomers. Smaller particles $(>10 \mathrm{~nm})$ have shorter circulation half lives due to renal clearance [2], and small size also limits the ability of the particle to be retained at the tumour site [3]. The Mn-porphysome's size makes it better suited for high yield delivery to the tumour. With the injection of the Mn-porphysome nanoparticle, we expect greater accumulation and therefore greater contrast enhancement of the tumour at both the 4 and 24 hour time points.

The subtle $\mathrm{T}_{1}$ effect at $4 \mathrm{H}$ may also be explained by a relatively small injected dose. Comparable liposome/porphyrin based manganese systems use an injected dose of $30 \mu \mathrm{mol} / \mathrm{kg}[4,5]$. To avoid potential toxicity effects observed in vitro, our system utilized a reduced dose. We anticipate once the toxicity issue is resolved and the intact nanoparticle is injected we will observe an even greater $T_{1}$ effect.

These preliminary results are validation of the ability of Mn-porphyrin-lipid to act as an MRI contrast agent for tumour detection. Toxicity must be addressed before a full dose can be explored. These results are promising evidence that nanoparticles constructed from these Mn-porphyrin-lipid monomers will be able to enhance tumour detection by MRI. 

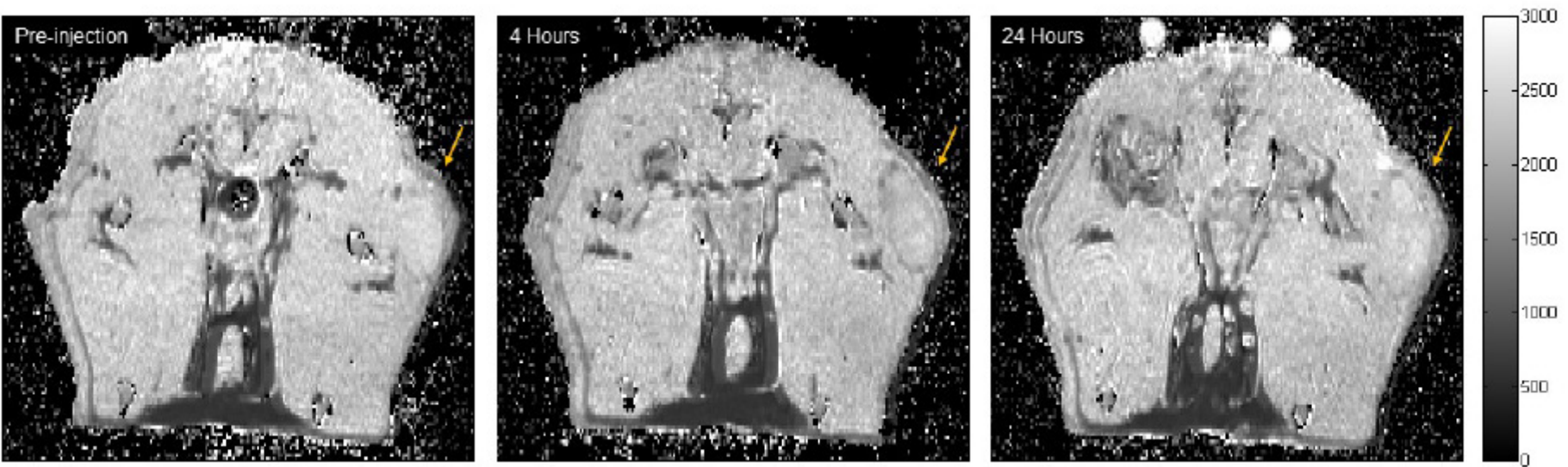

Supplementary Figure 1: $T_{1}$ relaxation map of subcutaneous tumour following Mn-porphyrin lipid injection.

Tumour site indicated with yellow arrow. Scale bar in ms. Left) Pre-injection. Centre) 4H post injection. Right) $24 \mathrm{H}$ post injection.
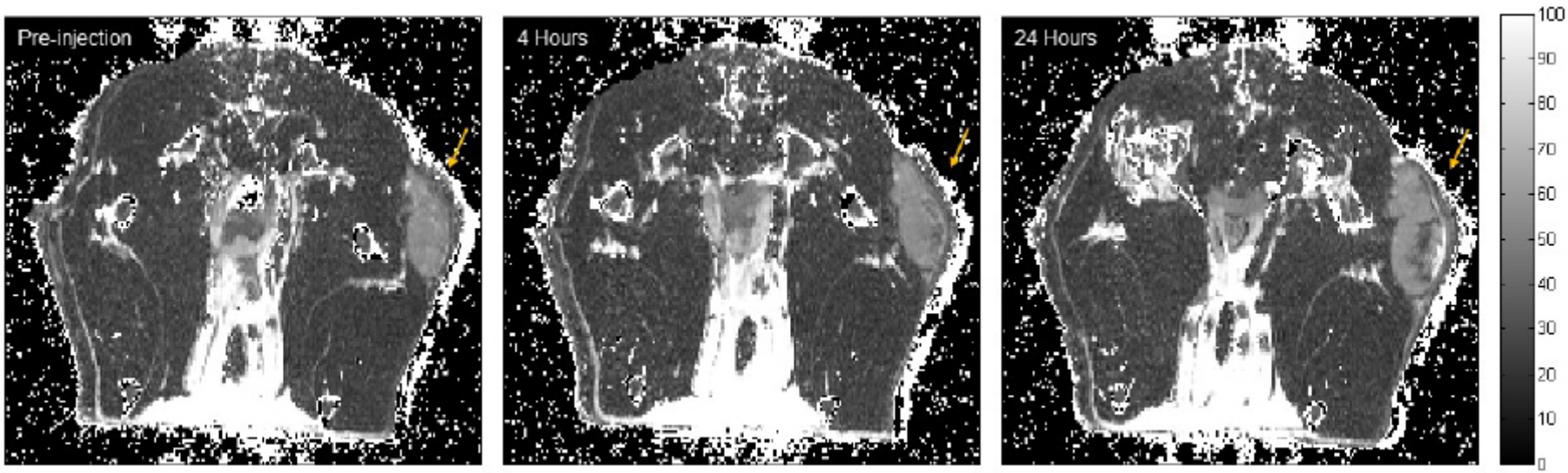

Supplementary Figure 2: $\mathrm{T}_{2}$ relaxation map of subcutaneous tumour following Mn-porphyrin lipid injection.

Tumour site indicated with yellow arrow. Scale bar in ms. Left) Pre-injection. Centre) 4H post injection. Right) 24H post injection.

\section{References}

1. Matsumura Y, Maeda H. A new concept for macromolecular therapeutics in cnacer chemotherapy: mechanism of tumoritropic accumulatio of proteins and the antitumor agents Smancs. Cancer Res. 1986;46(December):6387-92.

2. Choi HS, Liu W, Misra P, Tanaka E, Zimmer JP, Itty Ipe B, et al. Renal clearance of nanoparticles. Nat Biotechnol [Internet]. 2007;25(10):1165-70. Available from: http://dx.doi.org/10.1038/ nbt1340

3. Ernsting MJ, Murakami M, Roy A, Li SD. Factors controlling the pharmacokinetics, biodistribution and intratumoral penetration of nanoparticles. J Control Release. 2013;172(3):782-94.

4. Schwendener RA, W??thrich R, Duewell S, Westera G, von Schulthess GK. Small unilamellar liposomes as magnetic resonance contrast agents loaded with paramagnetic Mn-, Gd-, and Fe-DTPA-stearate complexes. Int J Pharm. 1989;49(3):249-59. 5. Kettritz U, Warshauer DM, Brown ED, Schlund JF, Eisenberg LB, Semelka RC. Enhancement of the normal pancreas: comparison of manganese-DPDP and gadolinium chelate. 1996;6(1):14-8. 